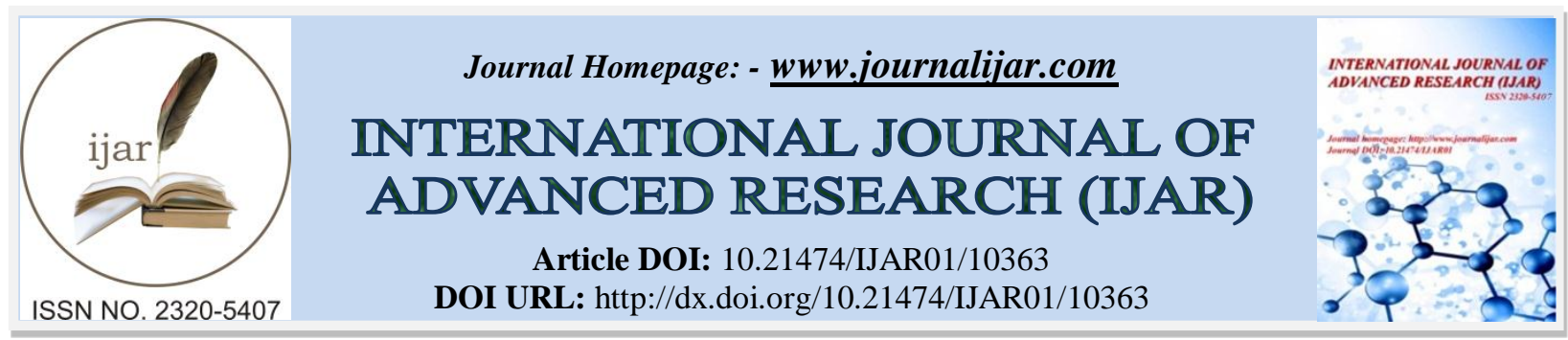

RESEARCH ARTICLE

\title{
PARTICULARITE DES SYNDROMES CORONARIEN AIGUE CHEZ LA FEMME, EXPERIENCE DU SERVICE DE CARDIOLOGIE DE L'HOPITAL MILITAIRE AVICENNE MARRAKECH
}

\author{
A. Chetoui, I. Benamar, L. Bendriss and A. Khatouri
}

Service De Cardiologie Et Maladies Vasculaires - Hôpital Militaire Avicenne De Marrakech.

\section{Manuscript Info}

\section{Manuscript History}

Received: 30 November 2019

Final Accepted: 31 December 2019

Published: January 2020

Key words:-

SCA, Maladie Coronaire, Femme, Coronarographie, Angioplastie

\section{Abstract}

La maladie coronaire est la première cause de mortalité chez la femme au niveau mondial. L'âge et la ménopause sont les facteurs de risque cardiovasculaires principaux chez la femme, avec à partir de 70 ans une prévalence identique dans les 2 sexes.

Matériel Et Méthode: Il s'agit d'une étude rétrospective descriptive comparative, portant sur une série de 116 cas répartis en deux groupes du même effectif (58 femmes et 58 hommes) ayant eu un syndrome coronarien aigu, colligée sur une durée de 5 ans, du $1^{\text {er }}$ janvier 2014 au 31 décembre 2018 menée au service cardiologie de l'Hôpital avicenne de marrakech.On a comparé dans notre étude les caractéristiques épidémiologiques, cliniques, agiographiques et les différences de prise en charge et de pronostic entre hommes et femmes face à la maladie coronaire, dans le cadre particulier de syndrome coronaire aigu.

Résultat: Les femmes étaient plus âgées, plus souvent hypertendues, diabétiques et moins souvent tabagiques que les hommes. Leur délai de prise en charge était plus long. Dans le groupe de femme, le SCA se présente dans $41 \%$ des cas sous forme SCA ST-, SCA ST+ (41\%), suivies de près de l'angor instable $(17 \%)$.Les femmes bénéficiaient moins souvent d'un traitement invasif et lorsqu'une coronarographie était réalisée, celle-ci retrouvait plus souvent des lésions relevant d'un traitement médical.

Conclusion: les résultats de notre étude vont nous inciter à modifier notre prise en charge du Syndrome coronaire aigu de la femme tout en tenant compte de leurs comorbidités et de leur Risque hémorragique. Sur le plan pratique. Il faut souligner l'importance de la préventionprimaire.

Copy Right, IJAR, 2020,. All rights reserved.

\section{Introduction:-}

La maladie coronaire chez la femme constitue un réel problème de santé publique. Contrairement à une opinion répandue, ce n'est pas le cancer du sein mais les maladies cardiovasculaires qui sont les premières causes de mortalité chez la femme [1]. Les résultats d'une étude épidémiologique récente, conduite en Europe, ont montrés que les cardiopathies ischémiques représentent à elles seules $22 \%$ des causes de décès chez la femme [1] [2].

Le but de cette étude est d'analyser les particularités épidémiologiques, cliniques et agiographiques de la coronaropathie chez la femme à travers une étude comparative entre deux groupes : Gp I (Femmes) et Gp II

Corresponding Author:- A. Chetoui

Address:- Service De Cardiologie Et Maladies Vasculaires - Hôpital Militaire Avicenne De Marrakech. 
(Hommes), analyser l'effet du sexe sur les facteurs de risque cardiovasculaire, les symptômes, et les résultats des examens réalisés ainsi que pourmettre en évidence les différences de prise en charge en fonction du sexe.

\section{Matériels et Methods:-}

Il s'agit d'une étude rétrospective comparative colligeant 116 observations tirées au hasard réparties en deux groupes: 58 femmes et 58 hommes, hospitalisés pour un syndrome coronarien aigue au Service de Cardiologie de l'hôpital militaire Avicenne(Marrakech), du Janvier 2014 à Décembre 2018.

A partir de l'analyse des dossiers médicaux nous avons rempli une fiche médicale contenant les données suivantes : les paramètres épidémio-cliniques, électro-cardiographiques, biologiques et le protocole thérapeutique à l'admission ainsi les résultats de la coronarographie, les modalités de la revascularisation myocardique et l'évolution hospitalière

Nous avons informatisé les données recueillis en utilisant le logiciel de statistique SPSS 0.2 à l'aide du centre d'épidémiologie de CHU. Ces données concernent : l'âge, les antécédents coronariens, les facteurs de risque cardiovasculaire, le mode de révélation de la maladie coronaire, les données de la coronarographie et les thérapeutiques entrepris. Les variables quantitatives sont exprimées en moyenne \pm écart-type et les variables qualitatives sont exprimées en effectif et pourcentage.

\section{Résultat:-}

L'incidence des SCA sur 1 an dans notre service chez les hommes était 8 fois supérieure que chez les femmes (20\%), la prévalence du sexe masculin est nettement supérieure. Les femmes sont âgées en moyen de 64,1+/- 9,1 ans avec des extrêmes de 47 à 89 ans par contre les hommes ont un âge moyen de $56+/-7,8$ ans et des extrêmes de 40 à 71 ans $(\mathrm{P}=0.002)$. L'âge représente un facteur de risques pour $75 \%$ des Femmes et 82,75\% des hommes $(\mathrm{P}=0.001)$. Le diabète représentant respectivement $41 \%$ dans le groupe I vs44, $8 \%$ dans le groupe II (P<0,05). Dans le groupe I l'HTA est présente chez 55,2\% des cas vs $27,6 \%$ des cas dans le groupe II ( $\mathrm{P}=0.033)$.L'obésité est présente chez $52,6 \%$ des femmes Vs16,7 \% dans le groupe des hommes $(\mathrm{P}=0.012)$. La dyslipidémie est notée dans le premier groupe chez $30,4 \%$ et dans le deuxième chez $39,1 \%(\mathrm{P}=0.536)$. La sédentarité est présente chez $50 \%$ des femmes Vs 3\% des hommes $(\mathrm{P}<0.001)$. Pas de notion de tabagisme dans notre série de femme vs41.4\% des hommes tabagique actif et $24,1 \%$ Sevrés. Trois pourcent des femmes ont une notion d'hérédité coronaire Vs $10,3 \%$ des hommes $(\mathrm{P}=0.333)$.

Tableau1:- Caractéristique de la douleur thoracique à l'admission et les signes accompagnateurs dans les 2 groupes.

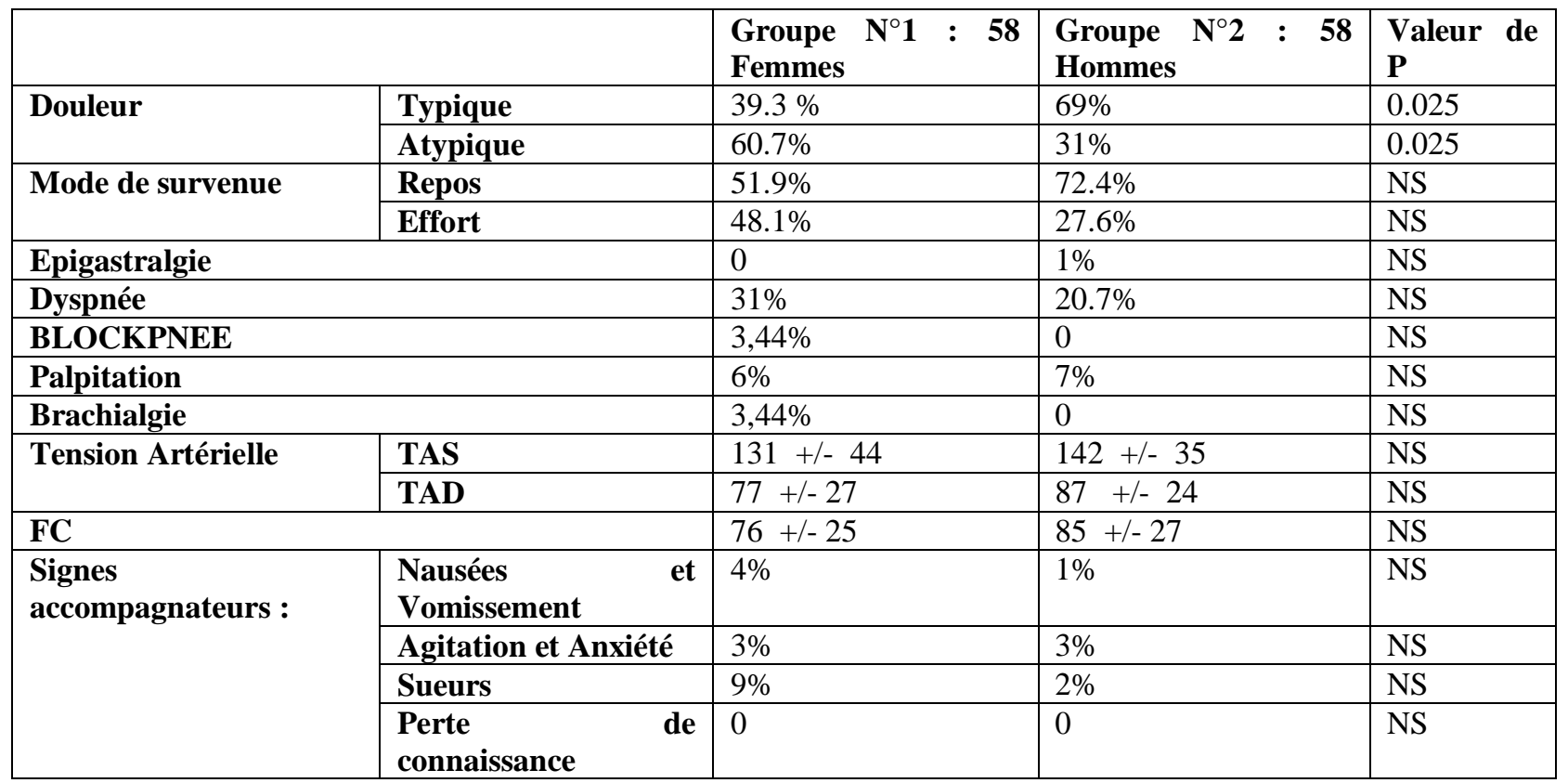


Dans notre série de femmes $81 \%$ d'entre elles sont ménopausées et $10,7 \%$ des femmes ont déjà une insuffisance rénale versus $6,9 \%$ chez les hommes $(\mathrm{P}=0,483)$. En comparaison aux hommes ,on a noté une nette comorbidité chez la femme : elle est plus âgée, ayant plus de diabète, de dyslipidémie, d'HTA, d'obésité et insuffisance rénale que chezl'homme.Sur le plan clinique les femmes décrivent plus souvent une douleur atypique que les hommes (60.7\% versus $31 \%$ ). Une douleur typique n'est retrouvée que chez $39.3 \%$ d'entre elles, contre $69 \%$ chez les hommes $(\mathrm{P}=0.025)$. En ce qui concerne le mode de survenue $51.9 \%$ des femmes représentent une douleur au repos contre $72.4 \%$ des hommes et $48.1 \%$ des femmes présentent une douleur à l'effort contre $27.6 \%$ des hommes $(\mathrm{P}=0.112)$. Les femmes présentent plus de signes accompagnateurs que leurs homologues masculins (tableau 1);et la blockpnée et la brachialgie ont été retrouvée chez 4 femmes Vs 0 homme (P non significatif).

Les caractéristiques biologiques ont démontré une morbidité féminine nette. Elles ont plus d'insuffisance rénale, d'anémie, de diabète décompensé et de syndrome inflammatoire biologique. Les hommes présentaient des taux plus élevés de marqueurs biologiques cardiaques.La prévalence NS La troponine était positive chez $72.4 \%$ des femmes versus $78.6 \%$ des hommes et négative : chez $27.6 \%$ des femmes Versus $21.4 \%$ des hommes ( $\mathrm{P}=0.021)$.

Sur le plan coronarographique les femmes présentaient des lésions plus serrées, plus longues et plus calcifiées que les hommes.L'atteinte monotronculaire était statiquement la plus fréquente chez les deux sexe cependant les femmes avaient tendance à présenter une atteinte polytronculaire $(21,1 \%)$ versus $(19 \%), \mathrm{P}=\mathrm{NS})$, En conclusion, la maladie coronaire chez la femme avait tendance à être plus étendue, plus complexe, caractérisée par une atteinte des gros troncs artériels épicardiques (tableau 2).

Tableau 2:- caractéristiques coronarographiques des deux groupe.

\begin{tabular}{|c|c|c|c|c|}
\hline \multicolumn{2}{|c|}{ 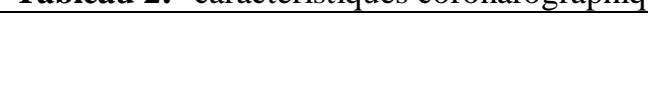 } & $\begin{array}{llll}\text { Groupe } & \mathbf{N}^{\circ} \mathbf{1} & \mathbf{5 8} \\
\text { Femmes } & & \end{array}$ & 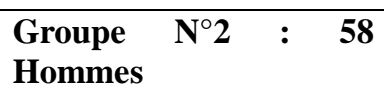 & $\begin{array}{l}\text { Valeur de } \\
\text { P }\end{array}$ \\
\hline \multicolumn{2}{|c|}{$\begin{array}{lll}\begin{array}{l}\text { Patients bénéficiant } \\
\text { coronarographie }\end{array} & \text { de } & \text { la } \\
\end{array}$} & $69 \%$ & $93 \%$ & 0.019 \\
\hline \multicolumn{2}{|c|}{ Coronarographie normale } & $5 \%$ & $3.7 \%$ & NS \\
\hline \multirow{3}{*}{$\begin{array}{l}\text { Nature de la } \\
\text { lésion }\end{array}$} & Athérome & $89.5 \%$ & $76.9 \%$ & NS \\
\hline & $\begin{array}{l}\text { Thrombose intra } \\
\text { stent }\end{array}$ & $5.3 \%$ & $11.5 \%$ & NS \\
\hline & Spasme & $5.3 \%$ & $11.5 \%$ & NS \\
\hline \multirow{3}{*}{$\begin{array}{l}\text { Nombre de } \\
\text { lésions }\end{array}$} & Monotroculaire & $42.1 \%$ & $46.2 \%$ & NS \\
\hline & Bitronculaire & $36.8 \%$ & $34.6 \%$ & NS \\
\hline & Tritronculaire & $21.1 \%$ & $19.2 \%$ & NS \\
\hline \multirow[t]{6}{*}{ Site de lésion } & IVA & $73.7 \%$ & $84.6 \%$ & NS \\
\hline & $\mathbf{C x}$ & $42.1 \%$ & $30.8 \%$ & NS \\
\hline & CD & $47.4 \%$ & $42.3 \%$ & NS \\
\hline & TC & $5.6 \%$ & $7.7 \%$ & NS \\
\hline & Marginale & $21.1 \%$ & $11.5 \%$ & NS \\
\hline & Diagonale & $10.5 \%$ & $7.7 \%$ & NS \\
\hline
\end{tabular}

\section{Aspects thérapeutiques et traitement medical:-}

Dans notre série la thrombolyse réussie a été réalisé chez 1 femme VS 2 hommes avec échec de la thrombolyse chez un homme ( $\mathrm{p}=\mathrm{NS})$. Au décours de la coronarographie, le traitement a été par angioplastie coronaire primaire chez $65 \%$ des femmes Vs $74 \%$ des hommes (Le stent actif a été réalisé chez 100\% des malades qui ont bénéficiés de l'angioplastie primaire),par pontage chez $10 \%$ des femmes Vs $7 \%$ des hommes et médical seul chez $44.8 \%$ des femmes Vs $31 \%$ des hommes.Pour les complications procédurale on a noté un hématome post coronarographique de la cuisse drainée avec transfusion de 4 culots globulaires chez une femme mais aucun décès per procédural n'a été enregistré.Tous les patients ont bénéficié d'un traitement antiischémique conventionnel incluant une héparinothérapie, du clopidogrél, de l'Aspirine, une statine et un bétabloquant ou inhibiteur calcique bradycardisant en cas de contre indications aux bétabloquants ou suspicion d'un vasospasme, un dérivé nitré.L'évolution au cours de l'hospitalisation a été marquée par la survenue dans les deux groupes de :Deux tachycardies ventriculaires ayant nécessité un choc électrique externe, un BAV deuxième degré transitoire chez les femmes et un cas de TV chez un homme.Deux cas d'insuffisances cardiaques gauches et deux globales dans le groupe 1 et Trois cas d'insuffisances cardiaques gauches une IVG Killip 3,1 cas d'insuffisance cardiaque globale et trois chocs cardiogéniques dans le 
groupe 2.Un thrombus avec ischémie du membre inférieure droit chez une femme et deux cas de thrombus intra VG chez deux hommes. Une infection nosocomiale àklebsiella pneumonie + hématome thoracique antérieur gauche chez une patiente en post pontage et deux cas d'acidocétoses diabétiques chez deux hommes.Pas de trouble mécanique noté dans les deux groupes.Un décès par choc cardiogénique chez une femme.

\section{Discussion:-}

La maladie coronaire est la première cause de mortalité chez la femme au niveau mondial. L'âge et la ménopause sont les facteurs de risque cardiovasculaires principaux chez la femme, avec à partir de 70 ans une prévalence identique dans les 2 sexes c'est pourquoi l'American Heart Association (AHA) et la Société européenne de cardiologie recommandent de dépister attentivement les femmes aux trois étapes clés de leur vie hormonale : la contraception, la grossesse et la ménopause[3,4].

Les différents travaux publiés s'intéressant aux disparités de genre dans le SCA ont constaté que les femmes rentrent dans la maladie coronaire 5 à 10 ans plus tard et qu'elles sont plus fréquemment hypertendues et diabétiques $[9,10,11]$. Les facteurs de risque que sont l'HTA et le diabète sont non seulement plus fréquents mais également à des niveaux de sévérité supérieurs, notamment pour le diabète [9,10,11], ce qui concorde avec notre résultat. Par contre, on n'apas noté de notion de tabagisme dans notre groupe de femme,mais dans la littérature les tendances mises en évidence dans FAST-MI sont assez alarmantes : si en 1995, elles étaient moins de $40 \%$ de femmes tabagiques actives, elles étaient plus de $70 \%$ en 2010[8].

Dans la littérature les comorbidités sont également plus fréquentes chez les femmes présentant un SCA comparativement à leurs homologues masculins.En effet, les femmes sont significativement plus âgées, elles ont une plus grande fréquence de diabète, ces deux facteurs influencent largement le pronostic de l'infarctus du myocarde [2].Dans notre étude on a une nette comorbidité chez la femme : elle est plus âgée, ayant plus de diabète, de dyslipidémie, d'HTA, d'obésité et insuffisance rénale que chezl'homme.

La présentation clinique dans la population féminine coronarienne présente de vraies différences avec la population masculine. Dans notre série, la prédominance significative de la symptomatologie atypique retrouvée chez la femme (60.7\% chez les femmes Vs $31 \%$ chez les hommes) concordait avec les données de la littérature.

Dans plusieurs études l'infarctus du myocarde ST positif apparaît en revanche moins fréquent chez la femme, ce qui laisserait à penser que la femme serait moins sujette à l'occlusion coronaire que l'homme .[17] Dans notre étude la femme a présenté un SCA ST+ seulement dans $41 \%$ versus $48 \%$ chez l'homme

L'infarctus du myocarde est dans l'inconscient collectif une pathologie essentiellement masculine. Ce doute diagnostique est aussi présent dans le corps médical et crée des hésitations dans la prise en charge. Une étude publiée par Pelletier et al. [12] montre chez des patients jeunes avec un SCA, des prises en charges très différentes en fonction du sexe. Les femmes avaient moins d'ECG réalisés dans les 10 minutes suivant 1 'admission (29 \% versus $38 \%, \mathrm{p}=0,02)$ et moins de chance d'avoir une stratégie de reperfusion coronaire $(83 \%$ versus $91 \%, \mathrm{p}=$ $0,01)[12]$

Le recours à l'angioplastie dans la stratégie de reperfusion est aussi significativement moins fréquent dans la population féminine et quelles que soit les tranches d'âges analysées Notre étude retrouve un taux de revascularisation par angioplastie plus faible chez les femmes que chez les hommes $(48.3 \%$ vs $69 \%$, p=NS)

Dans les études Prague 1 et 2, l'analyse de la mortalité dans le groupe transféré pour angioplastie en postthrombolyse montre un pronostic plus sombre dans la population féminine [8]. Dans le registre New-yorkais comprenant 11162 hommes et 2561 femmes (âgés de moins de 50 ans) bénéficiant d'une première angioplastie primaire dans le cadre du traitement d'un SCA avec sus-ST, le sexe féminin est un facteur de risque indépendant pour la mortalité hospitalière [9]. Pourtant, le succès d'angioplastie est comparable dans les deux sexes. Par ailleurs, les complications vasculaires sont significativement plus fréquentes chez les femmes $(0,82 \%$ versus $0,24 \%, \mathrm{p}<$ 0,0001).

Dans l'étude Cardio-ARHIF publiée en 2011 [12], la surmortalité dans la population féminine persistait malgré l'analyse multivariée permettant de s'affranchir des facteurs confondants cliniques et thérapeutique, d'évaluer le pronostic des patientes dans le cadre d'une prise en charge thérapeutique invasive puisque toutes les patientes ont 
fait l'objet d'une coronarographie et angioplastie si nécessaire. Cette surmortalité est d'ailleurs principalement observée en phase aiguë, lors de la prise en charge initiale et n'est plus retrouvée lors du suivi à 1 an [12].

Il est démontré que dans la population féminine, l'angioplastie en particulier dans la prise en charge des syndromes coronariens aigus, se complique plus fréquemment de complications hémorragiques [13] dans notre étude on a noté chez un hématome post coronarographique de la cuisse drainée avec transfusion de 4 culots globulaires chez une femme et aucun décès per procédural n'a été enregistré. À noter, que la voie d'abord radiale permet de réduire ce risque de complication en particulier pour les centres qui ont l'expérience de cette technique [13 ]. Notre étude retrouve une différence entre hommes et femmes concernant le taux de revascularisation par pontage aorto-coronaire (7\% chez les femmes vs $10.3 \%$ chez les hommes). Ces résultats sont concordants avec ceux du registre CRUSADE [16] qui retrouvait un taux de revascularisation par pontage aorto-coronaire plus faible chez les femmes $(9.0 \%$ vs $14.0 \%$ ) et avec ceux du registre GRACE [15] qui retrouvent des taux de recours au pontage similaires entre hommes et femmes .

\section{Conclusion:-}

Notre série a ainsi pu établir de manière significative que les femmes, bien que présentant un tableau clinique plus sévère que les hommes sont traitées de manière moins agressive. Néanmoins, les résultats de notre étude vont nous inciter à modifier notre prise en charge du syndrome coronaire aigu de la femme, ceci méritant une prise en charge plus agressive, tout en tenant compte de leurs comorbidités et de leur risque hémorragique. Sur le plan pratique, il faut conclure que devant la gravité de la pathologie, il est nécessaire de mettre en place une prise en charge complète et adaptée au profil de la patiente, offrant une efficacité maximale et une amélioration du pronostic. Il faut également souligner l'importance capitale de la prévention primaire afin de dépister l'ischémie myocardique, chez la femme en particulier.

\section{Bibliographies:-}

1. Nichols $\mathrm{m}$, townsend $\mathrm{n}$, scarborough $\mathrm{p}$, rayner $\mathrm{m}$.

2. European cardiovascular disease statistics 4th edition 2012 :euroheart ii. Eur Heart j $2013 ; 34: 3007$

3. Nichols $\mathrm{m}$, townsend $\mathrm{n}$, scarborough $\mathrm{p}$, rayner $\mathrm{m}$ Cardiovascular disease in europe 2014: epidemiological update.

4. Eur heart j. 2014 nov 7;35(42):2950-9. Doi: 10.1093/eurheartj/ehu299. Epub 2014 aug 19.

5. Aouba a et al.

6. Les causes médicales de décès en france en 2004 et leur évolution 1980-2004 (aouba@vesinet. Inserm.fr), centre d'épidémiologie sur les causes médicales de décès, inserm cépidc, le vésinet, france beh thématique 35$36 / 18$ septembre 2007. Mosca 1 et al.

7. For the american heart association. Aha guidelines. Circulation $2007 ; 115: 1481-501$

8. Ruiz-garcía j

9. Age- and gender-related changes in plaque composition in patients with acute coronary syndrome: the prospect study. Eurointervention. 2012 dec 20;8(8):929-38. Doi: 10.4244/eijv8i8a142

10. Bairey merz cn

11. Insights from the nhlbi-sponsored women's ischemia syndrome evaluation (wise) study: part ii: gender differences in presentation, diagnosis, and outcome with regard to gender-based pathophysiology of atherosclerosis and macrovascular and microvascular coronary disease. J am collcardiol. 2006 feb 7;47(3 suppl):s21-9. Reynolds hr1

12. Mechanisms of myocardial infarction in women without angiographically obstructive coronary artery disease.circulation. 2011 sep 27;124(13):1414-25. Doi: 10.1161/circulationaha.111.026542. Epub 2011 sep 6. Puymirat e

13. Association of changes in clinical characteristics and management with improvement in survival among patients with st-elevation myocardial infarction.

14. Jama. 2012 sep 12;308(10):998-1006. Doi: 10.1001/2012.jama.11348. 9-puymirat e Ii.

15. Association of changes in clinical characteristics and management with improvement in survival among patients with st-elevation myocardial infarction. 10-champney $\mathrm{kp}$

16. The joint contribution of sex, age and type of myocardial infarction on hospital mortality following acute myocardial infarction.heart. 2009 jun;95(11):895-9. Doi: 10.1136/hrt.2008.155804. Epub 2009 jan 15.11velders ma 
17. Influence of gender on ischemic times and outcomes after st-elevation myocardial infarction. Am $\mathrm{j}$ cardiol. 2013 feb 1;111(3):312-8. Doi: 10.1016/j.amjcard.2012.10.007. Epub 2012 nov 15. 12-benamer h1Iii.

18. Female gender is an independent predictor of in-hospital mortality after stemi in the era of primary pci: insights from the greater paris area pci registry.eurointervention. 2011 apr;6(9):1073-9. Doi: 10.4244/eijv6i9a187 13.de peretti c, chin f, tuppin p, danchin d. Personnes hospi- talisées

19. Pour infarctus du myocarde en france : tendances 2002-2008. Bull Epidemiolhebd 2012;41:459—65.

20. 15.dey s, flather md, devlin g, brieger d, et al.;

21. Global registry of acute coronary events investigators. Sex-related differences in the presentation, treatment and outcomes among patients with acute coronary syndromes: the global registry of acute coronary events. Heart. 2009 jan;95(1):20-6.16.blomkalns al, chen ay, hochman js, et al;

22. Crusade investigators. Gender disparities in the diagnosis and treatment of non-st-segment elevation acute coronary syndromes: large-scale observations from the crusade (can rapid risk stratification of unstable angina patients suppress adverse outcomes with early implementation of the american college of cardiology/american heart association guidelines) national quality improvement initiative. J am collcardiol. 2005;45(6):832-7. Iv. 17vaccarino $\mathrm{v}$

23. V. Sex-based differences in early mortality after myocardial infarction. National registry of myocardial infarction 2 participants. 1999. 